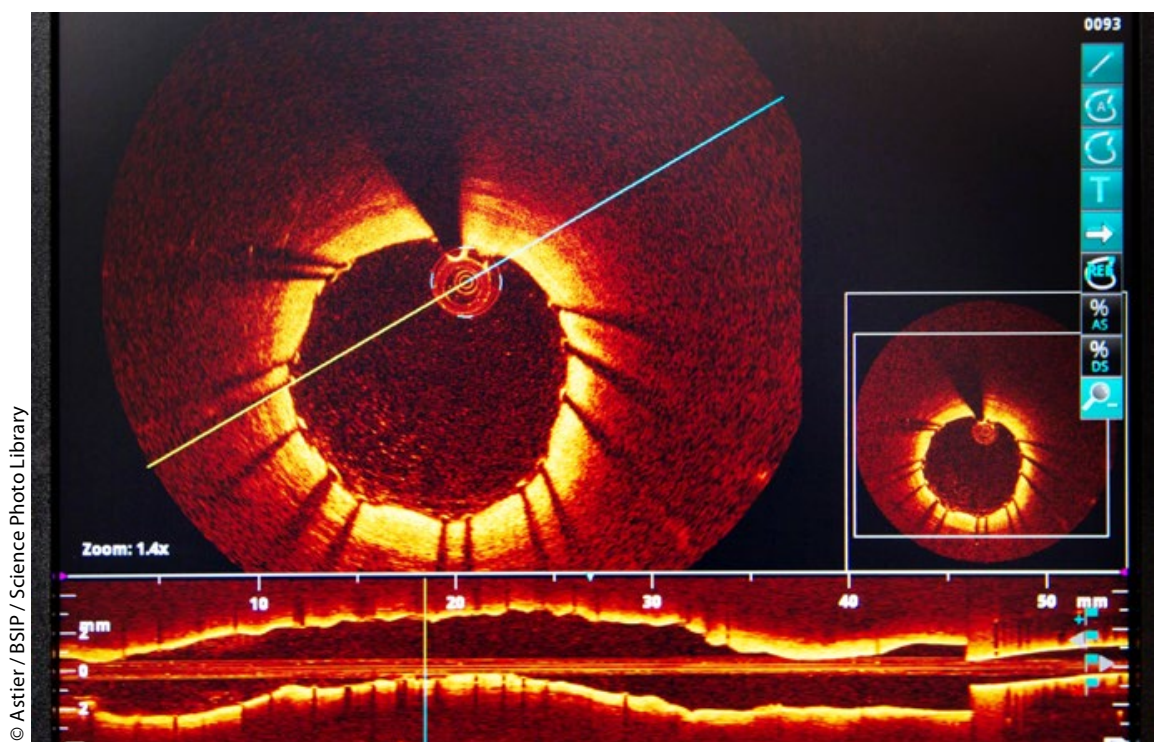

Mit der optischen Kohärenztomografie lässt sich - wie hier zu sehen - die Positionierung des Stents im Koronargefäß erkennen und kontrollieren.

\section{Scharfes Auge im Inneren von Koronargefäßen}

Die optische Kohärenztomografie (OCT) ist ein innovatives bildgebendes Verfahren in der invasiven KHK-Diagnostik. Sie liefert scharfe Bilder von der inneren Gefäßwand und deren Veränderungen. Diese Bildgebung könnte helfen, die KHK-Therapie stärker zu individualisieren.

$\mathrm{D}$ ie intrakoronare optische Kohärenztomografie (OCT) ermöglicht es, über einen kleinen Bildgebungskatheter und mithilfe von Infrarotlicht die koronare Gefäßwand sehr genau zu analysieren. Die räumliche Auflösung ist im Vergleich zum intravasalen Ultraschall ungleich höher. Damit lassen sich heute etwa atherosklerotische Veränderungen der Koronargefäße in Ausdehnung und Zusammensetzung sehr gut differenzieren, berichtete PD Dr. David M. Leistner von der Charité Berlin auf einer Pressekonferenz der DGK-Herztage in Berlin. Die dreidimensionale Darstellung liefert so wesentliche Zusatzinformationen zur Koronarangiografie, die bekanntlich nur ein Schattenbild des Gefäßlumens („Luminografie") zeichnen kann.

\section{Plaques besser differenzieren}

So kann nun besser zwischen lipidreichen Plaques, kalziumreichen Läsionen (Koronarkalk) und weichen „vulnerablen" Plaques, von denen aufgrund ihrer Rupturneigung die größte Gefahr aus- geht, unterschieden werden. Die OCT hilft zudem bei der Klärung der Gefäßpathologie im Falle eines akuten Koronarsyndroms. Damit lässt sich etwa zwischen Plaque-Ruptur und -Erosion unterscheiden, berichtete Leistner.

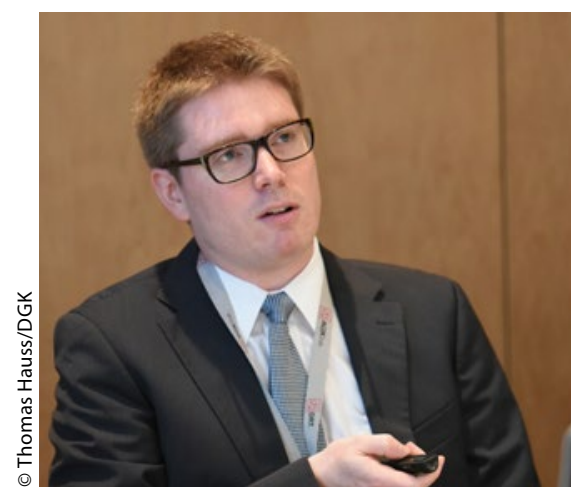

PD Dr. David M. Leistner von der Charité Berlin ist überzeugt von den Vorteilen der optischen Kohärenztomografie, mit der sich lipidreiche, kalziumreiche und weiche "vulnerable“ Plaques voneinander unterscheiden lassen.
Die OCT kann auch zur Verbesserung der Behandlungsergebnisse bei perkutaner koronarer Intervention (PCI) beitragen. Sie erleichtert die Entscheidung etwa über die optimale Stentlänge und ermöglichte es, die Stentplatzierung im Koronargefäß bezüglich Malapposition, Dissektion oder Gewebeprolaps durch Stentmaschen zu kontrollieren.

Dass dies von praktischer Bedeutung ist, zeigten, so Leistner, die Ergebnisse der ILUMIEN-1-Studie: Danach hatte die Verwendung der OCT-Bildgebung zur PCI-Optimierung wesentlichen Einfluss auf die Vorgehensweise vor und nach PCI.

\section{$\mathrm{PCl}$-Ergebnisse verbessert}

Vorteile der OCT bei akutem Koronarsyndrom konnten auch in der jüngst beim ESC-Kongress vorgestellten DOCTORS-Studie dokumentiert werden. Auch in dieser Studie, an der Patienten mit Nicht-ST-Hebungs-Myokardinfarkt (NSTEMI) beteiligt waren, hatte dieses Bildgebungsverfahren direkten Einfluss auf die ärztlichen Entscheidungen über die Vorgehensweise.

Im Ergebnis zeigte sich, dass dadurch die funktionellen Ergebnisse - gemessen an der fraktionellen Flussreserve (FFR) vor und nach PCI als primärem Endpunkt - im Vergleich zur Kontrollgruppe mit Koronarangiografie signifikant verbessert wurden. Ob dies am Ende auch in eine stärkere Reduktion von kardiovaskulären Ereignissen resultiert, werden größere Studien zeigen müssen.

\section{Personalisierte Medizin bei KHK?}

Die OCT könnte nach Ansicht Leistners auch die „Möglichkeiten für eine individualisierte Koronartherapie verbessern".

So wird derzeit unter anderem in Studien geprüft, ob bei Patienten mit akutem Herzinfarkt, bei denen die OCT Plaque-Erosionen als Ursache offenbart, auf eine Stentimplantation zugunsten einer reinen antithrombotischen Therapie verzichtet werden kann. Diese Erosionen heilen zumeist ohne Stenosierung wieder ab, gleichwohl werden auch diese $\mathrm{Pa}$ tienten heute routinemäßig mit einem Stent versorgt.

Peter Overbeck

Quelle: Pressekonferenz, DGK Herztage 2016, 06.10.2016, Berlin 